\title{
Frecuency of Do-Not-Resuscitate Orders in a Level 4 University Hospital
}

\section{Frecuencia de órdenes de no reanimación en un hospital universitario de cuarto nivel de complejidad}

\author{
Fritz Eduardo Gempeler Rueda ${ }^{\mathrm{a}}$ \\ Pontificia Universidad Javeriana, Colombia \\ Alejandra Sanín Hoyos \\ Pontificia Universidad Javeriana, Colombia \\ María de la Paz Echeverri L. \\ Hospital Universitario de San Ignacio, Colombia \\ Ángela lucía Balocco B. \\ Pontificia Universidad Javeriana, Colombia \\ Ana María Parra M. \\ Pontificia Universidad Javeriana, Colombia
}

a Correspondence: gempeler@javeriana.edu.co

How to cite: Gempeler Rueda FE, Sanín Hoyos A, Echeverri $M$ de la P, Balocco ÁL, Parra AM. Frecuency of Do-Not-Resuscitate Orders in a Level 4 University Hospital. Univ. Med. 2018;59(2). doi: https://doi.org/10.11144/Javeriana.umed59-2.rean

\begin{abstract}
After the introduction of the cardiopulmonary resuscitation into clinical practice, non-resuscitation orders emerged as an alternative for those patients that, for several reasons, were not candidates to receive this type of medical treatment. Over time, the non-resuscitation orders have increased in number, possibly due to the aging of the population, an increase in the prevalence of oncological pathologies or greater awareness of the physicians regarding the outcomes of the patients being carried to a CPR. According to the above, this study was developed with the objective of knowing and describing the frequency of DNR and CPR in patients who died in a level 4 hospital in the city of Bogotá. Likewise, the demographic characteristics of these patients were described.

Keywords

bioethics; cardiopulmonary resuscitation; resuscitation orders.
\end{abstract}

\section{RESUMEN}

Las órdenes de no reanimación (ONR) surgen luego de la introducción de la reanimación cardiopulmonar (RCP) en la práctica clínica como una alternativa para los pacientes que, por diferentes motivos, no eran candidatos para recibir este tipo de manejo médico. Con el tiempo se han incrementado las decisiones de no reanimar a los pacientes, posiblemente por el envejecimiento de la población, el aumento en la prevalencia de patologías oncológicas o una mayor sensibilización de los médicos en cuanto a los desenlaces de los pacientes que son llevados a una RCP. De acuerdo con lo anterior, se desarrolló este estudio con el objetivo de conocer y describir la frecuencia de las ONR y RCP en los pacientes que murieron en un hospital de cuarto nivel en la ciudad de Bogotá. Igualmente, se describieron las características demográficas de estos pacientes.

\section{Palabras clave}

Bioética; resucitación cardiopulmonar; órdenes de resucitación. 


\section{Introduction}

Shortly after the introduction of cardiopulmonary resuscitation (CPR) into clinical practice in 1960 , it became a mandatory treatment for all patients who had a cardiac arrest. Dying in a hospital meant going through CPR. One to two decades later, do-not-resuscitate (DNR) orders emerged in clinical practice, that is, the decision of not performing CPR maneuvers in patients who presented a cardiac arrest and had said order. This led to an essential change in medical management, because instead of indicating a specific treatment, what was sought was to refrain from intervening (1). Among the factors that influenced the development and the current adoption of DNR orders in the clinical practice are the low success rate of CPR (survival after hospital discharge ranging between $6.5 \%$ and $32 \%$ ), the growing theoretical and judicial development of the autonomy of individuals and of individual guarantees $(2,3,4,5,6)$.

DNR orders are defined today as decisions concerted between physicians and their patients or representatives not to attempt CPR in the event the patient suffers a cardiac arrest $(7,8)$. There will be patients who do not want CPR and others who wish to explore all the options and undergo multiple treatments, even with a poor quality of life, and this decision must be respected.

In recent years, the number of DNR orders has increased in patients dying in hospitals, ranging from $17 \%$ to $80 \%$ in different countries $(9,10,11,12)$. Although nowadays these orders are a common practice (1), no local studies have been conducted to establish its prevalence in patients hospitalized in a level 4 university hospital.

In order to know and describe the frequency of DNR orders and CPR in patients who died in said hospital, an observational-descriptive study was designed, based on the review of medical records; the study population were all patients who died in the hospital in 2015. The secondary objective of this study was to describe the demographic characteristics, DNR orders and CPR in the medical record and the participants in the DNR decision making.

The data were recorded in an Excel 2.1 database. For the quantitative variables, we described the frequencies of the events expressed in absolute numbers or percentages. When relevant, the averages with maximums and minimums and their respective dispersion measures were obtained.

\section{Results}

\section{General Information}

The protocol was approved by the Research Ethics Committee of the Hospital and the University. The study was carried out in a level 4 university hospital in Bogotá, Colombia, which has 440 beds and more than 18,000 hospitalizations per year (year 2015). Data of the deceased patients from January 1 to December 31, 2015 were obtained from the Department of Statistics.

\section{Characterization of the population}

In that period 832 patients died (Table 1), 51.5\% (428) of which were female, and 48.5\% (404), male. The average age at death was 60 years, with a range between 99 years and 1 hour of life. During that year, these patients were hospitalized an average of 10.5 days (range between 0 and 140 days) before dying.

Table 1.Deaths per Hospitalization Service

\begin{tabular}{|l|c|c|}
\hline \multicolumn{1}{|c|}{ Service } & Number of Deaths & $\%$ \\
\hline Emergency Service & 302 & 36.3 \\
\hline Intensive Care Unit & 206 & 24.8 \\
\hline Internal Medicine & 151 & 18.1 \\
\hline Surgery & 61 & 7.3 \\
\hline Pediatric and Neonatal Intensive Care Unit & 53 & 6.4 \\
\hline Gynecology & 28 & 3.4 \\
\hline Neuroscience & 16 & 1.9 \\
\hline Organ Donors & 9 & 1.1 \\
\hline Pediatric Service & 6 & 0.7 \\
\hline Total & 832 & 100.0 \\
\hline
\end{tabular}




\section{Main Diagnoses}

Of the 832 patients who died in 2015, 50.7\% (422 patients) had oncological pathologies within the main diagnoses, and 21.8\% (181 patients) had pneumonia as the final diagnosis. 1.92\% (16 patients) had brain death as the final diagnosis, but only 9 (1.08\% of the total of deaths) of these patients were organ donors.

\section{Do-not-resuscitate Orders}

70.9\% (590) of the deceased patients had a DNR order. Table 2 shows the distribution of these orders with respect to the total number of deaths per service.

Table 2.Distribution of Patients with a Do-not-resuscitate Order with Respect to the Total Number of Deaths per Service

\begin{tabular}{|l|c|c|c|}
\hline \multicolumn{1}{|c|}{ Service } & Number of Deaths & $\begin{array}{c}\text { DNR } \\
\text { orders }\end{array}$ & $\%$ \\
\hline Organ Donors & 9 & 9 & 100.00 \\
\hline Pediatric Service & 6 & 6 & 100.00 \\
\hline Gynecology & 28 & 25 & 89.29 \\
\hline Neuroscience & 16 & 14 & 87.50 \\
\hline Internal Medicine & 151 & 130 & 86.09 \\
\hline Surgery & 61 & 49 & 80.33 \\
\hline Emergency Service & 302 & 223 & 73.84 \\
\hline Intensive Care Unit & 206 & 120 & 58.25 \\
\hline Pediatric and Neonatal Intensive Care Unit & 53 & 14 & 26.42 \\
\hline Total & 832 & 590 & 70.91 \\
\hline
\end{tabular}

Patients with DNR orders had an average age of 64 years, and the patients without DNR orders had an average age of 50 years (DNR patients were 14 years older than those who did not have an order). Of the 590 DNR patients, $45 \%$ (265) were male, and 55\% (325) were female; $62.3 \%$ (368 patients) were oncological patients in terminal stages.

As can be seen in Table 3, 30.5\% (180) of the DNR decisions were of a medical nature, and were not informed to the patient or the family, or at least they were not described in the medical record, while $69.5 \%$ (410) of the decisions were established with the patient or with the family.
Table 3.Decision Making and Information about Do-notresuscitate Orders

\begin{tabular}{|l|c|c|}
\hline & Patients & $\%$ \\
\hline $\begin{array}{l}\text { Medical Decision not informed } \\
\text { to patient or family }\end{array}$ & 180 & 30.5 \\
\hline $\begin{array}{l}\text { Concerted Medical Decision, or } \\
\text { informed to patient or family }\end{array}$ & 410 & 69.5 \\
\hline Total & 590 & 100.0 \\
\hline
\end{tabular}

As can be seen in Table 4, most of the DNR orders informed to the patients or their families were established due to the patient's clinical condition, and only 5.1\% (21 patients) were due to advance directives.

Table 4.Concerted Medical Decision, or Informed to Patient or Family

\begin{tabular}{|l|c|c|}
\hline & Patients & $\%$ \\
\hline $\begin{array}{l}\text { Due to the patient's clinical } \\
\text { condition }\end{array}$ & 359 & 87.6 \\
\hline End-of-life Protocol & 30 & 7.3 \\
\hline Advances Directives & 21 & 5.1 \\
\hline Total & 410 & 100.0 \\
\hline
\end{tabular}

DNR orders were respected in $98.3 \%$ of the cases (in 580 patients), and only $1.7 \%$ of the DNR patients (10 patients) underwent CPR maneuvers despite what was recorded in the medical record. Of these 10 patients, 5 were in the Emergency service; 3 in the Intensive Care Unit, and 2 in the Internal Medicine service.

It should be noted that 44 patients $(5.28 \%$ of the patients who died) did not have a DNR order recorded in the medical record and did not undergo CPR. Table 5 shows the distribution of these patients per service. 
Fritz Eduardo Gempeler Rueda, Alejandra Sanín Hoyos, María de la Paz Echeverri L. et al.

Table 5.Distribution of Patients without a Do-not-resuscitate Order Who Underwent Cardiopulmonary Resuscitation per Service

\begin{tabular}{|l|c|c|c|}
\hline \multicolumn{1}{|c|}{ Service } & Number & $\begin{array}{c}\text { No DNR order } \\
\text { nor RCP }\end{array}$ & $\%$ \\
\hline Emergency Service & 302 & 21 & 6.95 \\
\hline ICU & 206 & 14 & 6.80 \\
\hline Internal Medicine & 151 & 3 & 1.99 \\
\hline Surgery & 61 & 3 & 4.92 \\
\hline $\begin{array}{l}\text { Pediatric and } \\
\text { Neonantal ICU }\end{array}$ & 53 & 3 & 5.66 \\
\hline Gynecology & 28 & 0 & 0.00 \\
\hline Neuroscience & 16 & 0 & 0.00 \\
\hline Organ Donors & 9 & 0 & 0.00 \\
\hline Pediatric Service & 6 & 0 & 0.00 \\
\hline Total & 832 & 44 & 5.29 \\
\hline
\end{tabular}

ICUIntensive Care Unit.

\section{Cardiopulmonary Resuscitation}

As can be seen in Table 6, 198 patients $(23.7 \%$ of the study population) underwent CPR before dying.

Table 6.Distribution of Patients Who Underwent Cardiopulmonary Resuscitation with Respect to the Total Number of Deaths per Service

\begin{tabular}{|l|c|c|c|}
\hline \multicolumn{1}{|c|}{ Service } & Number of Deaths & CPR & $\%$ \\
\hline Pediatric and Neonatal ICU & 53 & 36 & 67.92 \\
\hline ICU & 206 & 72 & 34.95 \\
\hline Emergency Service & 302 & 58 & 19.21 \\
\hline Surgery & 61 & 9 & 14.75 \\
\hline Neuroscience & 16 & 2 & 12.50 \\
\hline Internal Medicine & 151 & 18 & 11.92 \\
\hline Gynecology & 28 & 3 & 10.71 \\
\hline Organ Donors & 9 & 0 & 0.00 \\
\hline Pediatric Service & 6 & 0 & 0.00 \\
\hline Total & 832 & 198 & 23.80 \\
\hline
\end{tabular}

ICUIntensive Care Unit.

The descriptions of the CPR maneuvers recorded in the medical records (Table 7) were adequate in $77.8 \%$ (154 descriptions) of the cases, and they reported the time of the CPR maneuvers, medications used, doses, etc. On the other hand, $20.2 \%$ of the descriptions (40 cases) were incomplete. In 4 cases ( $2 \%$ of the CPRs) the physicians did not record the maneuvers performed; it was possible to know that CPR had been performed because of the nursing notes.

Table 7.Description of Cardiopulmonary Resuscitation in the Medical Record

\begin{tabular}{|l|c|c|}
\hline \multicolumn{1}{|c|}{ Cardiopulmonary Resucitation } & N & $\%$ \\
\hline Adequate & 154 & 77.8 \\
\hline Incomplete & 40 & 2.2 \\
\hline $\begin{array}{l}\text { The physician did not record the data in the } \\
\text { medical record }\end{array}$ & 4 & 2.0 \\
\hline Total & 198 & 100.0 \\
\hline
\end{tabular}

\section{Discussion}

This is the first study on the prevalence of DNR orders in patients of a level 4 university hospital in Colombia and on the characteristics of patients in the decision-making process that precedes the establishment of this type of status.

This study has some limitations. Since this is a retrospective study based solely on what was recorded in the medical records, it was not possible to know what doctors, patients or representatives thought when making the DNR decision. It was also not possible to determine the situations that may have influenced the punctual prevalence of the DNR decisions. Therefore, it only shows the facts, the frequency of the DNR orders and the data recorded in the medical records.

According to the results of this study, 7 out of 10 patients who died in the hospital had a DNR order; this shows that, in most cases, in this hospital it is not necessary to undergo CPR before to die. These decisions were based on the disease diagnosis and prognosis and, in large part, were taken during the course of the disease. Only 21 DNR orders (3.5\% of the 590 DNR orders) were established as advance directives.

The frequency of DNR orders in this study is higher than that of most other countries (Table 8). 
Table 8.Frequency of DNR Orders in Different Countries

\begin{tabular}{|l|c|c|}
\hline \multicolumn{1}{|c|}{ Country } & Frequency (\%) & References \\
\hline United States & 6 and 31 & $(1,13-14)$ \\
\hline Netherlands & 61 & $(15)$ \\
\hline United Kingdom & 80 & $(1)$ \\
\hline Belgium & 20.3 & $(16)$ \\
\hline Germany & 58 & $(17)$ \\
\hline
\end{tabular}

The results obtained (70.9\%) are significantly higher than those reported in the United States and Belgium, but similar to those of the United Kingdom and the Netherlands $(13,14,15,16,17,18,19,20,21)$. The frequency of DNRs varies not only from one country to another, but also between cities in the same country. Generally, DNRs are more frequent in patients with a short life expectancy and great limitations, but there are multiple factors that directly influence the variability in the frequency of DNRs. Patients, like their doctors, do not have the same perspectives and values about the end of life. Therefore, the differences in the frequency of DNRs may reflect, in part, the cultural differences and the preferences of hospitalized patients, or the differences in of the physicians' decisions according to their training and personal and religious opinions (22).

It is noteworthy that, although the percentage of DNRs in the different services exceeded $50 \%$, in the Pediatric Intensive Care Unit it reached only $26 \%$. The next service with a lower DNR frequency was the Adult Intensive Care Unit, with $58 \%$. In the rest of the services, the frequency of DNRs exceeded 70\%. In evaluating the distribution of DNRs with respect to the total number of deaths per service, it was found that the percentage was much lower in the intensive care units (adults, pediatric and neonatal). This may be due to the idea that the admission to an intensive care unit implies using all available therapeutic measures. In this way, it is possible that in these services the DNRs are established when all the hopes have been exhausted, and the deterioration of the patient is at a point of no return. It should be noted that 223 DNRs were established in the emergency service (73.8\% of patients who died in that service).
Although DNRs are not usually established in emergency situations unless the patient brings his/her written advance directive, this result could be explained by the presence of more than 70 observation beds that are frequently used by services such as Internal Medicine and Geriatrics, when there are no beds available in the respective services.

Some pathologies and characteristics of the patients that generate more DNRs have been described. Wachter et al. (23) reported that patients with AIDS and cancer were more likely to have a DNR order than patients with cirrhosis and heart failure, regardless of their life expectancy. Likewise, female patients older than 83 and patients with associated comorbidities such as depression and a poor prognosis were more likely to have a DNR order (23).

In the present study it was found that $62 \%$ of DNR orders were established in cancer patients, more in females than males $(55.1 \%$ vs. $44.9 \%)$. A relationship with age can also be seen, as DNR patients were older than those who did not have an order (64 years vs. 50 years). Although age alone could be a factor that influences the establishment of a DNR order, as suggested by Dautzenberg et al. (17), it has not been possible to demonstrate that it is an independent factor.

According to uncontrolled observations by the authors, DNR decisions are mostly late in the course of the disease, and the most important reasons for making these decisions are related to the severity of the disease and the poor prognosis. The short life expectancy and the disability do not always lead to the decision of establishing a DNR order. As can be seen, in the Neonatal Intensive Care Unit there were endstage patients without a DNR order, possibly due to the fact that they were neonates.

The high frequency of DNRs in the hospital could be explained by multiple reasons, such as the diagnoses (terminal cancer patients, among others) and the presence of trainee doctors. The fact that younger physicians are more likely to make this type of decisions could be due to a generational effect, due to the different attitudes or values instilled in medical schools, where patient autonomy is emphasized, as well as the 
respect for this autonomy. In addition, the efforts of the hospital's directives to integrate end-oflife care, supported by the Clinical Ethics Service, and the implementation of a DNR policy seem to lead physicians to reflect on the attribution of a DNR status to patients when appropriate.

Our results show that in $69.5 \%$ of the cases the non-resuscitation decision was taken with the patient or his/her relatives. This shows a greater interest in communicating this type of decisions and in including the patients' advance directives, as well as those who entered the end-of-life protocol. Even so, 30.5\% of DNR decisions, a significant percentage, was taken only by the physicians, without informing of them to the patient or his/her relatives, or at least this was not described in the medical record. Without exception, any medical order, such as a DNR order, must be recorded and explained in the medical record, and communicated to the patient and the medical and nursing team responsible for the patient's management. In this way doubts are avoided at times when acting immediately has a strong impact on the patient's morbidity and mortality. This could have prevented or diminished the possibility that patients with a DNR order were resuscitated, as happened in $1.7 \%$ of patients (10 cases).

According to the report by Van Delden et al. (15), in the Netherlands, one third of patients in the general services participated in the DNR decision, while in geriatric patients only $3 \%$ of patients and $24 \%$ of their families participated in these decisions (17). In the geriatric wards in Belgium, 4 out of 5 DNRs were discussed with the patients or their relatives (16). In Boston, United States, Murphy et al. found that the discussion on the DNR order with the patient was only documented in $2.8 \%$ of the 503 patients studied (24). As can be seen, there are large variations in the participation of the patients or their relatives in the DNR decision; this may be due to cultural differences, different doctor-patient relationships, different patterns of medical training, and the different personal and religious opinions (22).

The participation of patients or relatives in the DNR decision in the hospital was
$69.5 \%$. There are certain factors that could modify the participation of the patient or their relatives in the decision making process; one is the persistence of a paternalistic doctorpatient relationship, in which elderly patients are treated as if they were minors; another factor is the resistance of patients/relatives to discuss this issue, and leave the decision only to the doctor. The stress of talking about these issues with the patients/family, the patient's clinical condition and the determination of the patient's competence to make decisions could also influence the participation of patients and relatives.

Nowadays, it is widely recommended that in order to implement a DNR order, there should be a consultation between doctors and the patient or his/her representative. Patients should be informed more clearly and to a greater extent, and care must be taken to ensure that they have an active role, and they should be asked what they want to know $(4,25,26,27)$.

This study was based on what was described in the medical records; therefore, an additional factor could be that these interactions and their conclusions have not been adequately described in the medical record.

As mentioned, only 3.6\% (21 patients) had advance directives. Such a low number may be due to the lack of awareness of that alternative or to ignorance of the possibility of getting sick and dying at any time. Based on the results of this study, different intervention strategies can be proposed to optimize the DNR order and advance directive decision-making.

First, DNR orders must be discussed in advance, and the patients or their relatives should be included. It should never be a unilateral decision. Although CPR is not indicated from the medical point of view, this situation must be informed to the patient and his/ her family, and its justification must be explained and recorded in the medical record (16).

Secondly, information on advance directives, the possibility of death, the poor results of CPR, etc. should be disseminated in an optimal manner. Health personnel must have the knowledge, so that they can transmit this 
information optimally to patients and their families, so that lack of knowledge is not an obstacle to access.

It should be taken into account that DNR orders are not definitive, and that the patient can change his/her opinion about his/her clinical evolution at any time, and the doctor must be willing to listen and reach an agreement (28). It would be interesting to evaluate how these percentages change over time with the aforementioned interventions, and as the individual acquires a greater awareness as an integral being and the principles of autonomy and beneficence acquire greater importance (28).

Finally, our study allowed us to evaluate the quality of the descriptions of the CPR maneuvers performed by physicians in this institution. Noteworthy is that only $77.8 \%$ were considered complete, while $20.2 \%$ were incomplete; in a few cases, there was not even a record of that moment in the patient's medical record. It should not be forgotten that the medical record is a medicallegal document that arises between the health professional and the patient, where the care provided is recorded. If an appropriate record is not made, there will be no legal support to justify the reasons for the medical management, and that link with the patient will be broken.

Research on this subject and its derivatives should continue, especially in the field of DNR order and advance directive decision making.

\section{References}

1. Burns JP, Edwards J, Johnson J, Cassem NH, Truog RD. Do-not-resuscitate order after 25 years. Crit Care Med. 2003;31:1543-50.

2. Mohr M, Kettler D. Ethical aspects of resuscitation. Br J Anaesth. 1997;79:253-9.

3. McBrien M, Heyburn G. Do not attempt resuscitation orders in the peri-operative period. Anaesthesia. 2006;61:625-7.

4. Gempeler F. Reanimación cardiopulmonar más allá de la técnica. Rev Colomb Anestesiol. 2015;43:142-6.
5. Angus DC, Successful resuscitation from in-hospital cardiac arrest-What happens next? JAMA. 2015;314:1238-9.

6. Diem SJ, Lantos JD, Tulsky JA. Cardiopulmonary resuscitation on television: Miracles and misinformation. N Engl J Med 1996;334:1578-82.

7. Guarisco KK. Managing do-not-resuscitate orders in the peri anesthesia period. J Perianesth Nurs. 2004;19:300-7.

8. Van Norman G. Do-not-resuscitate orders during anaesthesia and urgent procedures [Internet]. 1998. Available from: http://depts.washington.edu/bioethx/ topics/dnrau.html.

9. Fritz Z, Fuld J. Ethical issues surrounding do not attempt resuscitation orders: decisions, discussion and deleterious effects. J Med Ethics. 2010;36:593-7.

10. Gan SC, Beaver SK, Houck PM. Treatment of acute myocardial infarction and 30-day mortality among women and men. N Engl J Med 2000;343:8-15.

11. Shepardson LB, Youngner SJ, Speroff T.Variation in the use of do-not-resuscitate orders in patients with stroke. Arch Intern Med. 1997;157:1841-7.

12. Phillips RS, Wenger NS, Teno J. Choices of seriously ill patients about cardiopulmonary resuscitation: Correlates and outcomes. Am J Med 1996;100:128-37.

13. Torian LV, Davidson EJ, Fillit HM, Fulop $G$, Sell LL. Decisions for and against resuscitation in an acute geriatric medicine unit serving the frail elderly. Arch Intern Med. 1992;152:561-5.

14. Wenger NS, Pearson ML, Desmond KA. Epidemiology of do-not-resuscitate orders: Disparity by age, diagnosis, gender, race, and functional impairment. Arch Intern Med 1995;155:2056-62.

15. Van Delden JJ, van der Maas PJ, Pijnenborg L, Looman CW. Deciding not to resuscitate in Dutch hospitals. J Med Ethics. 1993;19:200-5.

16. De Gendt C, Bilsen J, Van Den Noortgate N, Lambert M, Stichele RV, Deliens L. Prevalence of patients with do- 
not-resuscitate status on acute geriatric wards in Flanders, Belgium. J Gerontol. 2007;62A:395-9.

17. Dautzenberg PL, Duursma SA, Bezemer PD, Van Engen C, Schonwetter RS, Hooyer C. Resuscitation decisions on a Dutch geriatric ward. Q J Med. 1993;86:535-42.

18. De Vos R, Koster RW, de Haan RJ. Impact of survival probability, life expectancy, quality of life and patient preferences on do-notattempt-resuscitation orders in a hospital. Resuscitation Committee. Resuscitation. 1998;39:15-21.

19. Junod Perron N, Morabia A, De Torrente A. Evaluation of do not resuscitate orders (DNR) in a Swiss community hospital. J Med Ethics. 2002;28:36-7.

20. Youngner SJ, Lewandowski W, McClish DK, Juknialis BW, Coulton C, Bartlett ET. 'Do not resuscitate' orders. Incidence and implications in a medical-intensive care unit. JAMA. 1985;253:54-57.

21. Jayes RL, Zimmerman JE, Wagner DP, Draper EA, Knaus WA. Do-not-resuscitate orders in intensive care units. Current practices and recent changes. JAMA. 1993;270:2213-7.

22. Cherniack EP. Increasing use of DNR orders in the elderly worldwide: whose choice, is it? J Med Ethics. 2002;28:303-7.

23. Wachter RM, Luce JM, Hearst N, Decisions about resuscitation: inequities among patients with different diseases but similar prognoses. Ann Intern Med. 1989;111: 525-32.

24. Murphy DJ, Murray AM, Robinson BE, Campion EW. Outcomes of cardiopulmonary resuscitation in the elderly. Ann Intern Med. 1989;111:199-205.

25. Brindley PG. Perioperative do-not-resuscitate orders: It is time to talk. BMC Anesthesiology. 2013;13:1-3.

26. National Confidential Enquiry into Patient Outcome and Death (NCEPOD). Time to Intervene? A review of patients who underwent cardiopulmonary resuscitation as a result of an in- hospital cardiorespiratory arrest. Jun 2012 [cited 2014 May]. Available from: http://www.ncepod.org.uk/2012repor t1/downloads/CAPsummary.pdf.

27. Löfmark R, Nilstun T. Do-not-resuscitate orders-should the patient be informed? J Intern Med. 1997;241:1365-2796.

28. Gempeler FE, de Brigard-Pérez AM. Órdenes de no reanimación y anestesia. Rev Colomb Anestesiol. 2016;44:146-50. 